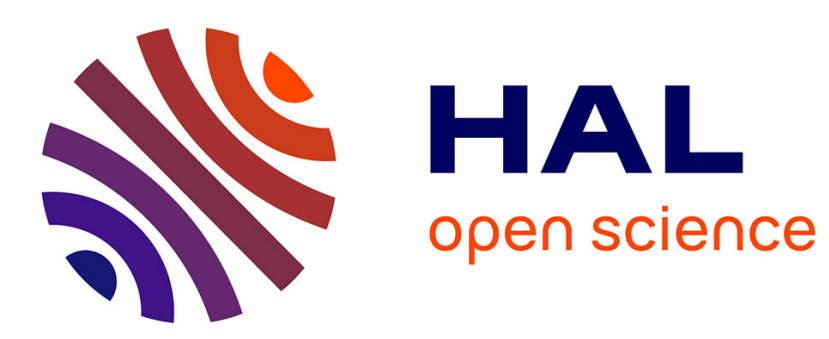

\title{
Open-ended coaxial probe: Model limitations
}

\author{
William Ellison, J.-M. Moreau
}

\section{To cite this version:}

William Ellison, J.-M. Moreau. Open-ended coaxial probe: Model limitations. IEEE Transactions on Instrumentation and Measurement, 2007. hal-00181023

\section{HAL Id: hal-00181023 \\ https://hal.science/hal-00181023}

Submitted on 24 Oct 2007

HAL is a multi-disciplinary open access archive for the deposit and dissemination of scientific research documents, whether they are published or not. The documents may come from teaching and research institutions in France or abroad, or from public or private research centers.
L'archive ouverte pluridisciplinaire HAL, est destinée au dépôt et à la diffusion de documents scientifiques de niveau recherche, publiés ou non, émanant des établissements d'enseignement et de recherche français ou étrangers, des laboratoires publics ou privés. 


\title{
Open-Ended Coaxial Probe: Model Limitations
}

\author{
William Ellison and Jacques-Marie Moreau
}

\begin{abstract}
We propose a procedure to determine the validity range of any empirical model of the open-ended coaxial probe transition that is used to measure the permittivity of materials. The procedure is illustrated by an application of the method to a standard coaxial cable probe, a permittivity range of interest in the food industry and, as an empirical model, the well-known "lumped capacitor model”.
\end{abstract}

Key wordss — Error analysis, Open-ended coaxial probe, Permittivity model

\section{INTRODUCTION}

The long history of the open-ended probe technique for the dielectric characterization of materials is reviewed in [1], [2], [4], [5], [6]. The method is widely used because it is simple to implement. It suffices to cut a wave-guide perpendicular to its axis, place the open end flush against the substance to be characterized and measure the admittance of the waveguide - material transition. The determination of the permittivity from this measurement is more delicate. The admittance, $Y(f, \varepsilon)$, is a relatively complicated function of: the frequency $f$, the permittivity $\varepsilon$ of the substance under investigation and the physical characteristics of the probe. However, at "low" frequencies the transition behaves like two simple capacitors in parallel and it has become customary to approximate the function $Y(f, \varepsilon)$ by the "lumped capacitor model": $Y(f, \varepsilon)=j 2 \pi f\left(C_{1}+\right.$ $C_{2} \varepsilon$ ), where $C_{1}, C_{2}$ are assumed to represent respectively the "line capacity" and the capacity of the transition "without the sample”. A common procedure is to assume that this model is valid for frequencies less than a few $\mathrm{GHz}$, that $C_{1}$ and $C_{2}$ do not depend upon $\varepsilon$ or $f$, and determine the constants $C_{1}, C_{2}$ by measuring the admittances of substances with known permittivities and then use the calibrated probe to measure substances with unknown $\varepsilon$.

Manuscript received November 25, 2005.

W. J. Ellison is with the I.M.S.. laboratory (Intégration du Matériau au Système), UMR-CNRS 5218., Ecole Nationale Supérieure de Chimie et de Physique de Bordeaux, 33607 Pessac, France (e-mail: william.ellison@enscpb.fr).

J. M. Moreau (retired) was with the P.I.O.M. laboratory, Ecole Nationale Supérieure de Chimie et de Physique de Bordeaux, 33607 Pessac, France. 
Surprisingly, the question of the validity of the model does not seem to have been systematically explored from the theoretical point of view. It is recognised that for a given probe this model is certainly not valid for "high" frequencies, but precisely what does "high” mean ? Is it 20 GHz, $10 \mathrm{GHz}, 5 \mathrm{GHz}, 1 \mathrm{GHz}$ or $100 \mathrm{MHz}$ ? The supposed precision of the values of $\varepsilon$ ' and $\varepsilon$ " cannot be seriously estimated without information about the precision of the model itself to represent the admittance function. The object of this article is to propose a procedure to determine, for a given probe and admittance model, frequency and permittivity ranges for which the model is valid to specified precision. As an example, we shall consider the case of the “lumped capacitor” model.

If we write $\varepsilon=\varepsilon^{\prime}-j \varepsilon^{\prime}$ and $Y(f, \varepsilon)=j 2 \pi f\left(C_{1}+\varepsilon C_{2}\right)$, then $\operatorname{Re}(Y)=2 \pi f C_{2} \varepsilon^{\prime \prime}$ and $\operatorname{Im}(Y)=2 \pi f\left(C_{1}+C_{2} \varepsilon^{\prime}\right)$. For a fixed $f$ and for fixed values of $C_{1}$ and $C_{2}$ the model induces a reticular parameterisation of the complex $Y$ plane as a function of $\varepsilon^{\prime}$ and $\varepsilon^{\prime}$, i.e. the contours for fixed $\varepsilon$ ' and variable $\varepsilon$ " are parallel horizontal straight lines and the contours for fixed $\varepsilon ”$ and variable $\varepsilon$ ' are parallel vertical stright lines. (See Fig. 1). Conversely, it follows that if the true contours of $\varepsilon^{\prime}$ and $\varepsilon$ ” in the $Y$ plane do not form a reticulation, then the simple capacitor model is certainly not valid for the given frequency and permittivity range.

The general idea of our method is to compare the contours induced in the complex admittance plane by the model with the ideal contours obtained by an exact calculation and then determine frequency and permittivity intervals for which the two reticulations differ by less than a specified amount (say 1\%).

\section{ANALYTIC EXPRESSIONS}

In certain idealised cases it is possible to calculate an exact analytical expression for $Y(f, \varepsilon)$ and so compute the corresponding contour grid. These cases are: a circular waveguide with an infinite ground-plane [5], [6], [7], a rectangular waveguide with an infinite ground-plane [10], [11], [12] and a coaxial waveguide with an infinite groundplane [8], [9], [4], [13], [15], [19].

We consider a perfectly conducting coaxial waveguide of internal radius $a$, external radius $b$, filled with a dielectric of permittivity $\varepsilon_{d}$ and possessing an infinite ground plane placed flush against a semi-infinite substance of permittivity $\varepsilon$ and permeability $\mu=1$. The expression for the admittance is:

$Y(f, \varepsilon)=\frac{v \varepsilon}{c \cdot \sqrt{\varepsilon_{d}} \ln (b / a)}\left(I_{0,0}+\sum_{n=1}^{\infty} \Lambda_{n} I_{0, n}\right)$

where the sequence $\left\{\Lambda_{n}: n=1,2, \ldots\right\}$ is the solution of the infinite system of linear equations:

$-I_{m, 0}=\sum_{n=1}^{\infty} A_{m, n} \Lambda_{n} \quad m=1,2,3, \ldots$

The coefficients $A_{m, n}$ of this system are given by

$$
A_{m, n}=I_{m, n}+\frac{2 \pi \varepsilon_{d} c \delta_{m, n}}{\varepsilon \sqrt{c^{2} k_{n}^{2}-4 \pi^{2} f^{2} \varepsilon_{d}}}
$$

where: the sequence $\left\{k_{n}: n=1,2,3, \ldots\right\}$ are the consecutive positive roots of the equation $J_{0}(a x) N_{0}(b x)=$ $N_{0}(a x) J_{0}(b x), \delta_{m, n}=1$ if $m=n$ and zero otherwise,

$$
I_{m, n}=\int_{a}^{b} \int_{a}^{b} \int_{0}^{2 \pi} \frac{\rho_{1} \rho_{2} R_{m}\left(\rho_{1}\right) R_{n}\left(\rho_{2}\right) \cos (\phi) e^{-j k_{0} r \sqrt{\varepsilon_{d}}} d \phi d \rho_{1} d \rho_{2}}{r},
$$


and $r^{2}=\rho_{1}^{2}+\rho_{2}^{2}-\rho_{1} \rho_{2} \cos (\phi)$

The functions $R_{n}(\rho)$ in the integrals (4) are defined by

$$
\begin{aligned}
& R_{0}(\rho)=\frac{1}{\rho \sqrt{\ln (b / a)}} \\
& R_{n}(\rho)=\frac{\pi k_{n} J_{0}\left(b k_{n}\right)\left(J_{1}\left(\rho k_{n}\right) N_{0}\left(a k_{n}\right)-N_{1}\left(\rho k_{n}\right) J_{0}\left(a k_{n}\right)\right)}{\sqrt{2\left(J_{0}^{2}\left(a k_{n}\right)-J_{0}^{2}\left(b k_{n}\right)\right)}}
\end{aligned}
$$

for $n=1,2, \ldots$

Thus, in order to calculate the admittance we must solve an infinite system of linear equations to determine the coefficients $\left\{\Lambda_{n}\right\}$. The infinite system (2) has to be truncated to a finite system. How many terms should be taken ? Various suggestions have been made, zero terms ([16], [17], [18]), three terms [19], six terms [20], twelve terms [21]. In reports from the National Physical Laboratory ([22], [23], [24]) comparative tests between various methods of calculation were made and the authors concluded that to have a satisfactory value of $Y(f, \varepsilon)$ one must use up to 50 terms! The number of terms that must be used depends upon many factors (probe characteristics, frequency and permittivity) and one cannot assign a fixed universal constant number. The computer programme should be arranged so as to increase this number automatically until the resulting calculated admittance has a predetermined tolerance.

The coefficients in the system of linear equations must be calculated very precisely. Errors in the values of $A_{m, n}$, together with an inappropriate truncation can lead to very inaccurate values for $\Lambda_{n}$ and hence for $Y(f, \varepsilon)$. The accurate numerical evaluation of the integrals $I_{m, n}$ posed a certain number of technical problems for a long time [8], [22],
[24]. The triple integrals $I_{m, n}$ have lines of singularities in the integrands for $\rho_{1}=\rho_{2}$ and $\varphi=0$ or $2 \pi$ and routine quadrature methods either gave incorrect results or consumed an inordinate amount of computer time.

One can transform the integrals (4) using Hankel transforms (see the Appendix for a sketch of the proofs and [6] for the details) into a more manageable form. It is convenient to introduce the following normalised variables:

$\beta=b / a, \quad \Omega=2 \pi f a \sqrt{ } \varepsilon_{d} / c, \quad q=\left(\varepsilon / \varepsilon_{d}\right)^{1 / 2}$

In terms of these variables, the integrals $I_{m, n}$ are:

$$
\begin{aligned}
& I_{0,0}=2 \pi a \int_{0}^{\infty} \frac{\left(J_{0}(\xi)-J_{0}(\beta \xi)\right)^{2}}{\xi \sqrt{\xi^{2}-(\Omega q)^{2}}} d \xi \\
& I_{0, n}=2 \pi a \int_{0}^{\infty} \frac{\xi\left(J_{0}(\xi)-J_{0}(\beta \xi)\right) F_{n}(\xi)}{\left(k_{n}^{2}-\xi^{2}\right) \sqrt{\xi^{2}-(\Omega q)^{2}}} d \xi \\
& I_{m, n}=2 \pi \int_{0}^{\infty} \frac{\xi^{3} F_{m}(\xi) F_{n}(\xi)}{\left(k_{n}^{2}-\xi^{2}\right)\left(k_{m}^{2}-\xi^{2}\right) \sqrt{\xi^{2}-(\Omega q)^{2}}} d \xi
\end{aligned}
$$

where $m \geq 1$ and $n \geq 1$ and $F_{n}(\xi)=J_{0}(\beta \xi) J_{0}\left(k_{n}\right)$ $J_{0}(\xi) J_{0}\left(\beta k_{n}\right)$.

The integrands have removable singularities at $\xi=0, \lambda_{n}$ and $\lambda_{m}$ respectively (If $\varepsilon$ is real, there is an an integrable singularity at $\xi=\Omega q$.). We write each of the infinite integrals as the sum of an intergral over the range $[0, X]$ plus an integral over the range $[X, \infty]$, where $X$ is "large", say, $X=\max \left\{100,2 / \Omega q /, 2 \lambda_{m}, 2 \lambda_{n}\right\}$. The integrals over the range $[0, X]$ contain the removable singularities, which now present no particular problems for the numerical integration. One must simply take care to correctly evaluate the integrands in the neighbourhood of the singular points. The integrals can be very quickly calculated to double 
precision by using a Gauss-Legendre or a Gauss-Kronrod Quadrature method [26], chapter 4.

For the integrals over the range $[X, \infty]$ the problem lies in the oscillatory nature of the integrands and the fact that they do not tend to zero very quickly. The basic idea is to replace the Bessel functions which occur in the integrands by asymptotic expansions with specific estimates for the error term. Watson [27], chapter 7, gives the following result:

For $x>0$ :

$$
J_{0}(x)=\sqrt{2 / \pi x}\left(\cos (x-\pi / 4) P_{n}(x)-\sin (x-\pi / 4) Q_{n}(x)\right.
$$

where for any $n \geq 1$

$$
\begin{gathered}
P_{n}(x)=1+\sum_{m=1}^{n} \frac{C_{2 m}(-1)^{m}}{x^{2 m}}+E_{1}(n, x) \\
Q_{n}(x)=\sum_{m=0}^{n} \frac{C_{2 m+1}(-1)^{m}}{x^{2 m+1}}+E_{2}(n, x) .
\end{gathered}
$$

The sequence $\left\{C_{r}\right\}$ is given for $r=0,1,2,3, \ldots$ by:

$C_{0}=1, C_{1}=1^{2} /\left(1 ! 8^{2}\right), \quad C_{r+1}=C_{r}(2 r+1)^{2} / 8(r+1)$

and

$$
\left|E_{1}(n, x)\right|<\frac{C_{2 n+2}}{x^{2 n+2}},\left|E_{2}(n, x)\right|<\frac{C_{2 n+3}}{x^{2 n+3}} .
$$

Using the above expansion the three types of integrals can be written as linear combinations of integrals of the kind

$$
\int_{1}^{\infty} \frac{\sin (X t)}{t^{r}} d t \quad \text { and } \quad \int_{1}^{\infty} \frac{\cos (X t)}{t^{r}} d t
$$

with an explicit error term. The integrals (15) can be quickly and accurately evaluated to double precision using a standard continued fraction algorithm, [26] chapter 6. The explicit nature of the error term (14) guarentees the precision of the calculated values of the intergrals.
We have implemented the above ideas in a PC program (Fortran + Visual Basic user interface). The program and its source code are freely available from the authors, together with the mathematical details of the algorithms that are used.

\section{A SPECIFIC EXAMPLE}

As an illustration of the procedure we consider the common $3.6 \mathrm{~mm}$ rigid coaxial line excited in the TEM mode. The relevant physical parameters of the line are: $a=$ $0.45925 \mathrm{~mm}, b=1.4925 \mathrm{~mm}, \varepsilon_{d}=2.15$. A permittivity range $5 \leq \varepsilon^{\prime} \leq 100,5 \leq \varepsilon^{\prime} \leq 100$ was used, since we are interested in humidity control testing. We calculated nomograms for $Y(f, \varepsilon)$ at 100, 10002000 and $5000 \mathrm{MHz}$. They are shown in Figs. 1, 2, 3, and 4. The admittances were calculated with a precision of \pm 0.001 . This involved trucating the infinite linear system (2) at up to 60 terms for some of the calculations. The horizontal lines correspond to constant values of $\varepsilon^{\prime}$. The bottom line is $\varepsilon^{\prime}=5$, the top line is $\varepsilon^{\prime}=100$, the increment is 5 units. The vertical lines correspond to constant values of $\varepsilon$ ". The far left line is $\varepsilon$ ” = 5, the far right line is $\varepsilon "=100$, the increment is 5 units. The departure from a square grid pattern is beginning to be visible at $2 \mathrm{GHz}$ and it is manifest at $5 \mathrm{GHz}$.

For any frequency $f$ less than $1 \mathrm{GHz}$ we have a square grid characterized by $C_{1}(f)$ and $C_{2}(f)$. To see whether $C_{1}$ and $C_{2}$ vary with frequency we calculated the grids for $f=0.1$, $0.2, \ldots, 1.0 \mathrm{GHz}$. The results are shown in Table I. We note that $C_{2}$ is practically constant and that $C_{1}$ varies by a factor 
of 3 over the frequency range 0.1 to $1.0 \mathrm{GHz}$

The variation of $C_{1}$ can be very closely represented by a quadratic function of $f: C_{1}(f)=a_{1}+a_{2} 4 \pi^{2} f^{2}$, where $a_{1}=$ 0.60102 and $a_{2}=-0.010670$, with a correlation coefficient of 0.999. Thus, even in conditions where the simple lumped capacitor model can be fitted to the exact admittance grid, the coefficients $C_{1}$ and $C_{2}$ vary with frequency. We note that several authors ([29], [30], [31]) in the course of careful experimental measurements have remarked that the parameters they found for the capacitor model varied with frequency and that this variation seemed to be quadratic. Thus, the variations which our theoretical calculations have revealed are perfectly detectable and should be taken into account. This means that instead of using the model $Y(f, \varepsilon)$ $=j 2 \pi f\left(C_{1}+C_{2} \varepsilon\right)$, where $C_{1}$ and $C_{2}$ do not depend upon $f$, one should use:

$Y(f, \varepsilon)=j 2 \pi f\left(A_{1}+A_{2} f^{2}+A_{3} \varepsilon\right)$,

or $\operatorname{Re}(Y)=-2 \pi f A_{3} \varepsilon^{\prime} \quad \operatorname{Im}(Y)=2 \pi f\left(A_{1}+A_{2}(2 \pi f)^{2}+A_{3} \varepsilon^{\prime}\right)$,

where the constants $A_{1}, A_{2}$ and $A_{3}$ do not depend upon $v$.

The $A_{i}$ can be determined by the calibration procedure discussed below.

One way of estimating the validity range of the model is to compute the percentage differences between the results of the exact calculation and the values of the admittance given by the model. Fig. 5 is such a representation for $f=1 \mathrm{GHz}$. The open squares correspond to those pairs $\left(\varepsilon^{\prime}, \varepsilon^{\prime \prime}\right)$ for which the difference is less than $1 \%$ and the solid squares to the pairs where the difference is greater than $1 \%$. If we use the interpolation for $C_{1}$ and $C_{2}$ given above, then the same precision holds for the same permittivity values over the frequency range 0 to $1 \mathrm{GHz}$. Thus, for permittivities $\left(\varepsilon^{\prime}, \varepsilon^{\prime}\right)$ within the area defined by the solid line in Fig. 5 and for frequencies between 0 and $1 \mathrm{GHz}$ the admittance model (16) can be calibrated so as to represent the theoretical admittance model with a difference of less than $1 \%$.

\section{PROBE CALIBRATION}

Why use the lumped capacity model at all? The exact calculation of the impedance will correspond to the measured impedance of a "real” probe if the hypotheses and numerical values assumed in the calculation correspond to the "real" probe. One could, in principle, use precalculated grids, such as Fig. 4, to read directly the permittivity from the measured admittance at any given frequency. Unfortunately real probes do not have infinite groundplanes, they are not perfect conductors, their linear dimensions are known with a relative error, perfect cylindricity and axiality are true up to a point, the central conductor is not exactly flush with the ground-plane etc. Variations in the calculated admittace due to an uncertainty in the physical charaaacteristics of the probe (such as the internal and external radii, $\mathrm{r}$ and $\mathrm{R}$, the permittivity of the coaxial cable filling etc.) are best estimated statistically by taking sequences of random values of the parameters $\mathrm{r}, \mathrm{R}$, $\varepsilon_{\mathrm{d}}$ within realistic error intervals and hen calculating the probbility distribution of the resulting values of the admittance. In precisely controlled laboratory conditions it is possible to use the exact calculations to determine 
absolute values of permittivity, but even so, the effect of the finite ground-plane is observed at certain resonant frequencies when measuring pure water [31]. Our concern is primarily with quality control in the food industry where the field conditions are far from being perfect and it is preferable to calibrate a given probe in terms of a specific empirical admittance model.

The admittance will usually be measured with a vector network analyser (VNA). The defects in the cables up to the connection with the coaxial probe will be taken into account by the manufactuers calibration kit and the measured admittance will be relative to this connection plane. However, what is required is the admittance at the probe/material interface at the end of the probe. There are three possibilities:

- a. If the probe is rigid, relatively short in length and in pristine condition, then one can suppose that there are no defects and the admittance at the probe/material interface can be calculated from the measured value at the VNA/probe connection using the electrical length of the probe to determine the phase change in the admittance at the end of the probe.

- b. Certain VNA's have a built-in time domain gating procedure that determines the admittance at the probe/material interface. The technique is attractive, but poses a certain number of subtle problems which are not easy to resolve [34], [35].

- c. If the time domain gating method is not available or the probe cannot be considered perfect, then it is usual to model the defects along the line with a two port transfer matrix. The transfer matrix combined with the lumped capacity model yields: $Y(f, \varepsilon)=j 2 \pi f\left(D_{1}+\right.$ $\varepsilon D_{2}$ ), where $D_{1}$ and $D_{2}$ are complex numbers which depend upon $f$.

In order to calibrate the probe we must work in frequency and permittivity ranges for which the lumped capacity model differs by say $1 \%$ from the theoretical calculation and use reference media with permittivities within the valid range to determine either the coefficients $a_{1}, a_{2}, a_{3}$ or the coefficients $D_{1}(f)$ and $D_{2}(f)$.

If we are in cases (a) or (b) then $a_{1}, a_{2}, a_{3}$ can be determined uniquely from the imaginary part of the admittance and a knowledge of $\varepsilon^{\prime}$ of the reference media. This observation is important because the number of references substances is rather limited and for calibration purposes one should cover the desired permittivity range. It is easy to prepare water-alcohol solutions or saline solutions that have $\varepsilon^{\prime}$ at any given value in the range 10 to 80 by an appropriate mixture. Precise permittivity data for these solutions over a wide temperature range are readily available ([36], [37], [38], [39], [40]).

\section{CONCLUSION}

The open-ended coaxial probe is frequently used to chrcterise the dielectric properties of maaterials via admittance measurements. The deduction of the permittiviy of the material from the admittance data is made by assuming that the admittance of the probe/materiaal 
interface is described by some simple empirical model. Such models are not universally valid. We propose a method to determine the frequence range an permittivity intervals for which a given model has at most a specified deviation from an exact theoretical calculation of the interface admittance. As an illustration of the general method we apply it to the commly used open-ended coaxial probe and the "lumped capacity" model.

\section{APPENDIX}

The transformation of the integrals (4) to the integrals (10) is not new, but we have not found an easily accessible selfcontained exposition and the derivation is not obvious without some guidelines. We refer to a standard treatise for specific theorems and explicitly indicate the more routine mathematical operations.

$\underline{\text { Lemma } 1}$ If $z \in C, b \geq 0$ and if either $\operatorname{Re}(\mathrm{z})>0$ and $b \geq 0$ or $\operatorname{Re}(\mathrm{z})=0$ and $b \neq \operatorname{Im}(\mathrm{z})$ then

$\int_{0}^{\infty} e^{-z \lambda} J_{0}(b \lambda) d \lambda=\left(z^{2}+b^{2}\right)^{-0.5}$

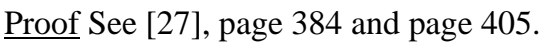

Lemma 2 If $\operatorname{Re}(a)>0, R>0$ then

$\frac{e^{-a R}}{R}=\int_{0}^{\infty} \frac{\lambda \cdot J_{0}(R \cdot \lambda)}{\sqrt{\lambda^{2}+a^{2}}} \cdot d \lambda$

Proof Use Hänkel's double integral theorem [27], chapter

14:

If the integral $\int_{0}^{\infty} F(r) \sqrt{r} d r$ exists and is absolutely

convergent, then

$$
F(R)=\int_{0}^{\infty} \lambda J_{0}(\lambda R) \int_{0}^{\infty} r F(r) J_{0}(\lambda r) d r d \lambda
$$

with $F(R)=e^{-a R} / R$ and use lemma 1 .

Lemma 3 If $\operatorname{Re}(a)>0, R^{2}=\rho_{1}{ }^{2}+\rho_{2}{ }^{2}-\rho_{1} \rho_{2} \cos (\phi)$ then,

$\int_{0}^{2 \pi} \frac{e^{-a R}}{R} \cos (\varphi) d \varphi=2 \pi \int_{0}^{\infty} \frac{\lambda J_{1}\left(\lambda \rho_{1}\right) J_{1}\left(\lambda \rho_{2}\right)}{\sqrt{\lambda^{2}+a^{2}}} d \lambda$

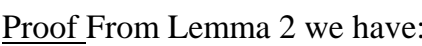

$$
\begin{aligned}
& \mathrm{I}=\int_{0}^{2 \pi} \frac{e^{-a R}}{R} \cos (\varphi) d \varphi=\int_{0}^{2 \pi} \int_{0}^{\infty} \frac{2 \lambda J_{0}(R \lambda)}{\sqrt{\lambda^{2}+a^{2}}} \cos (\varphi) d \lambda d \varphi \\
& =\int_{0}^{\infty} \frac{\lambda}{\sqrt{\lambda^{2}+a^{2}}} \int_{0}^{2 \pi} J_{0}(R \lambda) \cos (\varphi) d \varphi d \lambda
\end{aligned}
$$

We now apply Neumann's addition theorem [27] , Chapter 11: "If $R^{2}=\rho_{1}^{2}+\rho_{2}^{2}-\rho_{1} \rho_{2} \cos (\phi)$ then, $J_{0}(R \lambda)=\sum_{n=0}^{\infty}\left(2-\delta_{0, n}\right) J_{n}\left(\lambda \rho_{1}\right) J_{n}\left(\lambda \rho_{2}\right) \cos (n \varphi) "$

Substitute the above expression for $J_{0}(R \lambda)$ in the final integral to obtain:

$\int_{0}^{\infty} \frac{\lambda}{\sqrt{\lambda^{2}+a^{2}}} \int_{0}^{2 \pi} \sum_{n=0}^{\infty}\left(2-\delta_{0, n}\right) J_{n}\left(\lambda \rho_{1}\right) J_{n}\left(\lambda \rho_{2}\right) \cos (n \varphi) \cos (\varphi) d \varphi d \lambda$

$=$

$\int_{0}^{\infty} \frac{\lambda}{\sqrt{\lambda^{2}+a^{2}}} \sum_{n=0}^{\infty}\left(2-\delta_{0, n}\right) J_{n}\left(\lambda \rho_{1}\right) J_{n}\left(\lambda \rho_{2}\right) \int_{0}^{2 \pi} \cos (n \varphi) \cos (\varphi) d \varphi d \lambda$

Since $\int_{0}^{2 \pi} \cos (n \varphi) \cos (\varphi) d \varphi$ is equal to $\pi$ if $n=1$ and is zero otherwise we obtain the stated result.

Lemma 4 If $\operatorname{Re}(\kappa)>0$ and $R^{2}=\rho_{1}^{2}+\rho_{2}^{2}-\rho_{1} \rho_{2} \cos (\phi)$ then

$\mathrm{I}_{0,0}=\int_{a}^{b} \int_{a}^{b} \int_{0}^{2 \pi} \frac{e^{-\kappa R}}{R} \cos (\varphi) d \varphi d \rho_{1} d \rho_{2}=2 \pi \int_{0}^{\infty} \frac{\left\{J_{0}(a \lambda)-J_{0}(b \lambda)\right\}^{2}}{\lambda \sqrt{\lambda^{2}+\kappa^{2}}} d \lambda$

$\underline{\text { Proof }}$

$\mathrm{I}_{0,0}=\int_{a}^{b} \int_{a}^{b} \int_{0}^{2 \pi} \frac{e^{-\kappa R}}{R} \cos (\varphi) d \varphi d \rho_{1} d \rho_{2}$ $=2 \pi \int_{a}^{b} \int_{a}^{b} \int_{0}^{\infty} \frac{\lambda J_{1}\left(\lambda \rho_{1}\right) J_{1}\left(\lambda \rho_{2}\right)}{\sqrt{\lambda^{2}+\kappa^{2}}} d \lambda d \rho_{1} d \rho_{2}$ 


$$
=2 \pi \int_{0}^{\infty} \frac{\lambda}{\sqrt{\lambda^{2}+\kappa^{2}}} \int_{a}^{b} J_{1}\left(\lambda \rho_{1}\right) d \rho_{1} \int_{a}^{b} J_{1}\left(\lambda \rho_{2}\right) d \rho_{2} d \lambda
$$

Recall that $\frac{d J_{0}(x)}{d x}=-J_{0}(x)$ and so $\int_{a}^{b} J_{1}(x) d x=J_{0}(a)-J_{0}(b)$.

Thus

$\int_{a}^{b} J_{1}\left(\lambda \rho_{1}\right) d \rho_{1}=\frac{\left\{J_{0}(a \lambda)-J_{0}(b \lambda)\right\}}{\lambda}$ and $\int_{a}^{b} J_{1}\left(\lambda \rho_{2}\right) d \rho_{2}=\frac{\left\{J_{0}(a \lambda)-J_{0}(b \lambda)\right\}}{\lambda}$,

which gives the result.

Lemma 5 If $\lambda \neq \lambda_{n}$ then the following indefinite integrals hold:

$\int \rho J_{1}(\lambda \rho) J_{1}\left(\lambda_{n} \rho\right) d \rho=\frac{\rho}{\lambda_{n}^{2}-\lambda^{2}}\left\{\lambda J_{1}\left(\lambda_{n} \rho\right) J_{0}(\lambda \rho)-\lambda_{n} J_{0}\left(\lambda_{n} \rho\right) J_{1}(\lambda \rho)\right\}$

$\int \rho J_{1}(\lambda \rho) N_{1}\left(\lambda_{n} \rho\right) d \rho=\frac{\rho}{\lambda_{n}^{2}-\lambda^{2}}\left\{\lambda N_{1}\left(\lambda_{n} \rho\right) J_{0}(\lambda \rho)-\lambda_{n} J_{0}\left(\lambda_{n} \rho\right) N_{1}(\lambda \rho)\right\}$

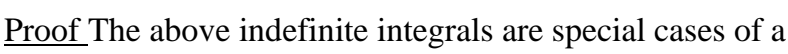
general formula for Cylinder functions, [27], Chapter 5, equation (8). The formula in question is:

If $\mathrm{C}_{\mu}(z)$ and $\overline{\mathrm{C}_{\mu}(z)}$ are any two Cylinder functions of order $\mu$ and $k \neq \ell$, then

$\int z \mathrm{C}_{\mu}(\mathrm{kz}) \overline{\mathrm{C}_{\mu}(\mathrm{Iz})} \cdot d z=\frac{z}{k^{2}-\mathrm{I}^{2}}\left\{k \mathrm{C}_{\mu+1}(k z) \overline{\mathrm{C}_{\mu}(\mathrm{Iz})}-\mathrm{IC}{ }_{\mu}(k z) \overline{\mathrm{C}_{\mu+1}(\mathrm{Iz})}\right\}$

From [26], page 82, we have, for all Cylinder functions, the relation:

$$
\mathrm{C}_{\mu-1}(z)+\mathrm{C}_{\mu+1}(z)=\frac{2 \mu}{z} \mathrm{C}_{\mu}(z)
$$

If we use this relation to eliminate $\mathrm{C}_{\mu+1}(z)$ and $\overline{\mathrm{C}_{\mu+1}(\mathrm{z})}$ from the above expression we obtain, after algebraic manipulation, the following expression for the indefinite integral:

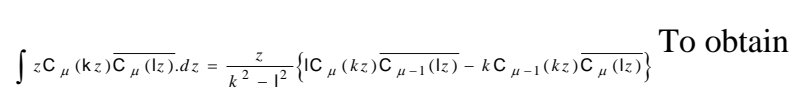

(1), take $z=\rho, k=\lambda, \ell=\lambda_{n}, C_{\mu}=J_{1}, \overline{\mathrm{C}_{\mu}}=J_{1}$.

To obtain (2) take $\mathrm{z}=\rho, k=\lambda, \ell=\lambda_{n}, \mathrm{C}_{\mu}=J_{1}, \overline{\mathrm{C}_{\mu}}=N_{1}$.

$\underline{\text { Lemma } 6}$ If $n \geq 1$ and $R^{2}=\rho_{1}^{2}+\rho_{2}^{2}-\rho_{1} \rho_{2} \cos (\phi)$, then

$$
\mathrm{I}_{0, n}=\int_{a}^{b} \int_{a}^{b} \rho_{2} R_{n}\left(\rho_{2}\right) \int_{0}^{2 \pi} \frac{e^{-\kappa R}}{R} \cos (\varphi) d \varphi d \rho_{1} d \rho_{2}
$$

$$
=\frac{4}{\lambda_{n} J_{0}\left(\lambda_{n} b\right)} \int_{0}^{\infty} \frac{\lambda\left\{J_{0}(\lambda a)-J_{0}(\lambda b)\right\}}{\left(\lambda_{n}^{2}-\lambda^{2}\right) \sqrt{\lambda^{2}+\kappa^{2}}} F_{n}(\lambda) d \lambda
$$

where $F_{n}(\lambda)=C_{n} \cdot\left\{J_{0}(\lambda b) J_{0}\left(\lambda_{n} \cdot a\right)-J_{0}\left(\lambda_{n} b\right) J_{0}(\lambda . a)\right\}$

$\underline{\text { Proof }}$

$$
\begin{aligned}
& \mathrm{I}_{0, n}=\int_{a}^{b} \int_{a}^{b} \rho_{2} R_{n}\left(\rho_{2}\right) \int_{0}^{2 \pi} \frac{e^{-\kappa R}}{R} \cos (\varphi) d \varphi d \rho_{2} d \rho_{1} \\
& =2 \pi \int_{a}^{b} \int_{a}^{b} \rho_{2} R_{n}\left(\rho_{2}\right) \int_{0}^{\infty} \frac{\lambda J_{1}\left(\lambda \rho_{1}\right) J_{1}\left(\lambda \rho_{2}\right)}{\sqrt{\lambda^{2}+\kappa^{2}}} d \lambda d \rho_{2} d \rho_{1} \\
& =2 \pi \int_{0}^{\infty} \frac{\lambda}{\sqrt{\lambda^{2}+a^{2}}} \int_{a}^{b} \rho_{2} \cdot R_{n}\left(\rho_{2}\right) \cdot J_{1}\left(\lambda \rho_{2}\right) \cdot d \rho_{2} \cdot \int_{a}^{b} J_{1}\left(\lambda \rho_{1}\right) \cdot d \rho_{1} \cdot d \lambda \\
& =2 \pi \int_{0}^{\infty} \frac{\lambda}{\sqrt{\lambda^{2}+a^{2}}} \int_{a}^{b} \rho_{2} R_{n}\left(\rho_{2}\right) J_{1}\left(\lambda \rho_{2}\right) d \rho_{2} \frac{\left\{J_{0}(\lambda a)-J_{0}(\lambda b)\right\}}{\lambda} d \lambda \\
& =2 \pi \int_{0}^{\infty} \frac{\left\{J_{0}(\lambda a)-J_{0}(\lambda b)\right\}}{\sqrt{\lambda^{2}+a^{2}}} \int_{a}^{b} \rho_{2} R_{n}\left(\rho_{2}\right) J_{1}\left(\lambda \rho_{2}\right) d \rho_{2} \cdot d \lambda
\end{aligned}
$$

$=2 \pi \cdot C_{n} \int_{0}^{\infty} \frac{\left\{J_{0}(\lambda a)-J_{0}(\lambda b)\right\}}{\sqrt{\lambda^{2}+a^{2}}} \int_{a}^{b} \rho_{2} J_{1}\left(\lambda \rho_{2}\right)\left\{J_{1}\left(\lambda_{n} \rho_{2}\right) N_{0}\left(\lambda_{n} a\right)-J_{0}\left(\lambda_{n} a\right) N_{1}\left(\lambda_{n} \rho_{2}\right)\right\} d \rho_{2} d \lambda$

We now have to evaluate the integral:

$\int_{a}^{b} \rho_{2} J_{1}\left(\lambda \rho_{2}\right)\left\{J_{1}\left(\lambda_{n} \rho_{2}\right) N_{0}\left(\lambda_{n} a\right)-J_{0}\left(\lambda_{n} a\right) N_{1}\left(\lambda_{n} \rho_{2}\right)\right\} d \rho_{2}$.

It is equal to:

$N_{0}\left(\lambda_{n} a\right) \int_{a}^{b} \rho_{2} J_{1}\left(\lambda \rho_{2}\right) J_{1}\left(\lambda_{n} \rho_{2}\right) d \rho_{2}-J_{0}\left(\lambda_{n} a\right) \int_{a}^{b} \rho_{2} J_{1}\left(\lambda_{n} \rho_{2}\right) N_{1}\left(\lambda_{n} \rho_{2}\right) d \rho_{2}$

The integrals: 
$\int_{a}^{b} \rho_{2} J_{1}\left(\lambda \rho_{2}\right) J_{1}\left(\lambda_{n} \rho_{2}\right) d \rho_{2}$ and $\int_{a}^{b} \rho_{2} J_{1}\left(\lambda \rho_{2}\right) N_{1}\left(\lambda_{n} \rho_{2}\right) d \rho_{2}$

have been evaluated in Lemma 5. If we substitute the expressions we find that the integral is equal to:

$\frac{\lambda b}{\lambda_{n}^{2}-\lambda^{2}}\left[J_{1}\left(\lambda_{n} b\right) J_{0}(\lambda b) N_{0}\left(\lambda_{n} a\right)-N_{1}\left(\lambda_{n} b\right) J_{0}(\lambda b) J_{0}\left(\lambda_{n} a\right)\right]$

$-\frac{\lambda_{n} b}{\lambda_{n}^{2}-\lambda^{2}}\left[J_{0}\left(\lambda_{n} b\right) J_{1}(\lambda b) N_{0}\left(\lambda_{n} a\right)-N_{0}\left(\lambda_{n} b\right) J_{1}(\lambda b) J_{0}\left(\lambda_{n} a\right)\right]$

$-\frac{\lambda_{n} a}{\lambda_{n}^{2}-\lambda^{2}}\left[J_{1}\left(\lambda_{n} a\right) J_{0}(\lambda a) N_{0}\left(\lambda_{n} a\right)-N_{1}\left(\lambda_{n} a\right) J_{0}(\lambda b) J_{0}\left(\lambda_{n} a\right)\right]$

The term in the second bracket is equal to zero because the quantities $\lambda_{n}$ are defined so that $J_{0}\left(\lambda_{n} b\right) . N_{0}\left(\lambda_{n} a\right)=$

$N_{0}\left(\lambda_{n} b\right) . J_{0}\left(\lambda_{n} a\right)$. We now use the relation: $J_{1}(\mathrm{z}) N_{0}(\mathrm{z})-$

$J_{0}(z) N_{1}(z)=2 / \pi z$, taking in turn $z=\lambda_{n} b$ and $z=\lambda_{n} a$, to

eliminate $N_{1}\left(\lambda_{n} b\right)$ and $N_{1}\left(\lambda_{n} a\right)$ from the first and third

brackets. After a simple algebraic manipulation we arrive at the following expression for the integral:

$\frac{2}{\pi \lambda_{n} J_{0}\left(\lambda_{n} b\right)} \cdot \frac{\lambda}{\lambda_{n}^{2}-\lambda^{2}}\left\{J_{0}(\lambda a) J_{0}\left(\lambda_{n} b\right)-J_{0}(\lambda b) J_{0}\left(\lambda_{n} a\right)\right\}$

Substituting this expression in the integral we obtain the stated result.

Lemma 7 If $m>0, n>0, \operatorname{Re}(\kappa)>0, R^{2}=\rho_{1}^{2}+\rho_{2}^{2}-$ $\rho_{1} \rho_{2} \cos (\phi)$ then

$$
\begin{aligned}
I_{m, n} & =\int_{a}^{b} \int_{a}^{b} \int_{0}^{2 \pi} \rho_{1} R_{m}\left(\rho_{1}\right) \rho_{2} R_{n}\left(\rho_{2}\right) \frac{e^{-\kappa R}}{R} \cos (\varphi) d \varphi d \rho_{1} d \rho_{2} \\
& =\frac{8}{\pi \lambda_{m} \lambda_{n} J_{0}\left(\lambda_{m} b\right) J_{0}\left(\lambda_{n} b\right)} \int_{0}^{\infty} \frac{\lambda^{3} F_{m}(\lambda) F_{n}(\lambda)}{\left(\lambda_{m}^{2}-\lambda^{2}\right)\left(\lambda_{n}^{2}-\lambda^{2}\right) \sqrt{\lambda^{2}+\kappa^{2}}} d \lambda
\end{aligned}
$$

Proof By Lemma 3 we have:

$$
\begin{aligned}
& \mathrm{I}_{m, n}=2 \pi \int_{a}^{b} \rho_{1} R_{m}\left(\rho_{1}\right) \int_{a}^{b} \rho_{2} R_{n}\left(\rho_{2}\right) \int_{0}^{\infty} \frac{\lambda J_{1}\left(\lambda \rho_{1}\right) J_{1}\left(\lambda \rho_{2}\right)}{\sqrt{\lambda^{2}+\kappa^{2}}} d \lambda d \rho_{1} d \rho_{2} \\
& =2 \pi \int_{0}^{\infty} \frac{\lambda}{\sqrt{\lambda^{2}+\kappa^{2}}} \int_{a}^{b} \rho_{1} R_{m}\left(\rho_{1}\right) J_{1}\left(\lambda \rho_{1}\right) d \rho_{1} \int_{a}^{b} \rho_{2} R_{n}\left(\rho_{2}\right) J_{1}\left(\lambda \rho_{2}\right) d \rho_{2} d \lambda
\end{aligned}
$$

In the proof of Lemma 6 we showed that:

$\int_{a}^{b} \rho R_{m}(\rho) J_{1}(\lambda \rho) d \rho=\frac{2}{\pi \lambda_{m} J_{0}\left(\lambda_{m} b\right)} \cdot \frac{\lambda F_{m}(\lambda)}{\lambda_{m}^{2}-\lambda^{2}}$

Replacing the integrals with respect to $\rho_{1}$ and $\rho_{2}$ by the corresponding expressions gives the stated result.

\section{REFERENCES}

[1] M. Stuchly, S. Stuchly, "Coaxial line reflection methods for measuring dielectric properties of biological substances at radio and microwave frequencies - A Review”, IEEE Trans. Instrum. Meas., vol. 29, pp. 176-183, 1980.

[2] F. E. Gardiol, “Open-ended waveguides: Principles and applications", Advances in Electronics and Electron Physics, vol. 63, Ed. P. Hawkes, Academic Press, New York 1985.

[3] C. L. Pournaropoulos, D. Misra, "The coaxial aperture electromagnetic sensor and its application in material characterization”, Meas. Sci. Techno. vol. 8, pp. 1191-1202, 1997.

[4] M. Gex-Fabry, J. Mosig, F. E. Gardiol, “ Reflection and radiation from an open-ended circular waveguide: Applications to nondestructive measurement of materials”, Arch. Elek. Ubertragung, vol. 33, pp. 473-478, 1979.

[5] F. E. Gardiol, T. Sphicopoulos, V. Teodoridis, "The reflection of open-ended circular wave-guides," in Reviews of Infrared and Millmeter Waves vol. 1, New York, Plenum Press, pp. 225 - 264, 1983.

[6] W. J. Ellison, J-M. Moreau, 'Theory and practice of open-ended dielectric probes”, unpublished notes.

[7] C. Fray, N. Khayata, A. Papiernik, "TM01 admittance and radiation from a flanged open-ended waveguide into layered absorbing media”, Arch. Elek. Ubertragung.., vol. 36, pp. 107-110, 1982.

[8] K. Bois, A. Benatly, R. Zoughi, "Multimode solution for the reflection properties of an open-ended rectangular waveguide radiating into a dielectric halfspace.”, IEEE Trans. Instrum. Meas., vol. 48, pp. 1131-1140, 1999.

[9] C. W. Chang, K. M. Chen, J. Qian, "Nondestructive determination of electromagnetic parameters of dielectric materials at X-band 
frequencies using a waveguide probe system”, IEEE Trans. Instrum. Meas., vol. 46, pp. 1084-1092, 1997.

[10] M. C. Decreton, M. S. Ramachandraiah, "Nondestructive measurement of complex permittivity for dielectric slabs”, IEEE Trans Micro. Theory Tech., vol. 23, pp. 1077-1080, 1975.

[11] H. Levine, C. Papas, "Theory of the circular diffraction antenna”, $J$. of Applied Physics vol. vol. 22, pp. 29-43, 1951.

[12] J. Mosig, J. Besson, M. Gex-Fabry, F. E. Gardiol, "Reflection of an open-ended coaxial line and application to nondestructive measurement of materials”, IEEE Trans. Instrum. Meas. vol. 30, pp. 46-51, 1981.

[13] G. Gajda, S. Stuchly, “An equivalent circuit of an open-ended coaxial line”, IEEE Trans. Instrum. Meas., vol. 32, pp. 506-508, 1983.

[14] Z. Delecki, S. Stuchly, “Analysis of the open-ended coaxial line sensors”, J. Electromag. Waves and Appli., vol 4, pp. 169-183, 1990.

[15] C. L. Li, K. M. Chen, "Determination of electromagnetic properties of materials using flanged open ended coaxial probe - Full wave analysis”, IEEE Trans. Instrum. Meas. vol. 44, pp. 19-27, 1995.

[16] Y. Noh, H. Eom, "Radiation from a flanged coaxial line into a dielectric slab.” IEEE Trans. Instrum. Meas. vol. 49, pp. 2158-2161, 1999.

[17] D. Misra, “A quasistatic analysis of open ended coaxial lines.”, IEEE Trans. Microwave Theory Tech., vol. 35, pp. 925-928, 1987.

[18] D. Misra, M. Chabbra, B. Epstein, M. Mirotznik, K. Foster, "Noninvasive electrical characterization of materials at microwave frequencies using an open-ended coaxial line: Test of an improved calibration technique”, IEEE Trans. Microwave Theory and Tech. vol. 38, pp. 8-14, 1990.

[19] D. V. Blackham, R. D. Pollard, “An improved technique for permittivity measurements using a coaxial probe.”, IEEE Trans. Instrum. Meas. vol. 46, pp. 1093-1099.

[20] L. Li, N. Ismail, L. Taylor, C. Davis, "Flanged coaxial microwave probes for measuring thin moisture layers”, IEEE Trans. Biomed. Engin., vol 38, pp.49-57, 1991.

[32] probes in dielectric spectroscopy”, Phys. Med. Biol. vol. 39, pp. 2183-2200, 1994.
[21] J. Baker-Jarvis, M. Janezic, P. Domich, R. Geyer, “Analysis of an open ended coaxial probe with lift-off for non-destructive testing”, IEEE Trans. Instrum. Meas., vol. 43, pp. 711-718, 1994.

[22] J. Baker-Jarvis, M. Janezec, C. Jones, "Shielded open-circuited sample holder for dielectric measurements of solids and liquids”, IEEE Trans. Instrum. Meas., vol. 47, pp. 338-344, 1998.

[23] A. Warham, “Annular slot antenna radiating into lossy material”, N.P.L. Report DITC 152/89, November 1989.

[24] S. Jenkins, A. Preece, T. Hodgetts,, G. T. Symm, A. Warham, R. Clarke, "Comparison of three numerical treatments for the openended coaxial line sensor”, Electronic Letters, vol. 26, pp.234-236, 1990.

[25] R. Clarke, A. Gregory, T. Hodgetts, "Improvements in coaxial sensor dielectric measurements”, Microwave Aquametry, Ed. A. Kraszewski, pp. 279-297, IEEE Press (1996).

[26] W. H. Press, S. A. Teukolsky, W. T. Vetterling, B. P. Flannery, Numerical recipes in Fortran: The art of scientific computing, C.U.P., Cambridge, 1992.

[27] G. N. Watson, A treatise on the theory of Bessel functions, $2^{\text {nd }}$ edition, C.U.P., Cambridge 1980.

[28] C. Li, K. Chen, "Determination of electromagnetic properties of materials using flanged open-ended probes - Full wave analysis”, IEEE Trans. Instrum. Meas., vol. 44, pp. 1887-1889, 1997.

[29] A. Kraszewski, S. Stuchly, "Capacitance of open-ended dielectric filled coaxial lines: Experimental results”, IEEE Trans. Instrum. Meas., vol. 32, pp. 517-519 1983.

[30] T. W. Athey, M. A. Stuchly and S. Stuchly, "Measurement of radio frequency permittivity of biological tissues with an open-ended coaxial line, part 1”, IEEE Trans. Microwave Theory Tech., vol. 30, pp. 82-86, 1982

[31] C. Gabriel, T. Y. A. Chan, E. H. Grant, “Admittance models for open ended coaxial

[33] S. Jenkins, T. Hodgetts, R. Clarke, A. Preece, "Dielectric measurement on reference liquids using automatic network analysers 
and calculable geometries”, Meas. Sci. Technol. vol.1, pp. 691-702, 1990.

[34] S. Jenkins, A. Warham, R. Clarke, "Use of open-ended coaxial line sensor with a liminar or liquid dielectric backed by a conducting plane”, IEEE Proceedings-H., vol. 139, pp. 179-182, 1991.

[35] R. De Porrata-Doria, A. B. Ibars, "Analysis and reduction of the distortion induced by time domain filtering techniques in network analysers”, IEEE Trans. Instrum. Meas. vol 47, pp. 930-934, 1998.

[36] P. M. T. Broersen, "Facts and fiction in spectral analysis", IEEE Trans. Instrum. Meas., vol. 49, pp. 766-772, 2000.

[37] S. Evans, S. Michelson, "Intercomparison of dielectric reference materials available for the calibration of an open-ended probe at different temperatures”, Meas. Sci. Technol. vol. 6, pp. 1721-1732, 1995.

[38] S. Mashimo, S. Yagihara, K. Higasi, "The dielectric relaxation of mixtures of water and primary alcohol”, J. Chem. Phys. vol. 90, pp.3292-3294, 1989.

[39] D. Bertolini, M. Cassettari, G. Salvetti, "The dielectric properties of alcohol-water solutions. I The alcohol rich region”, J. Chem. Phys. vol. 78, pp. 365-372, 1983.

[40] S. Evans, S. C. Michelson, "Intercomparison of dielectric reference materials available for the calibration of an open-ended probe at different temperatures”, Meas. Sci. Technol. vol. 6, pp. 1721-1732, 1995.

[41] N. L. Buck, “Calibration of dielectric constant probes using salt solutions of unknown conductivity”, IEEE Trans. Instrum. Meas., vol. 45, pp.84-88, 1996.

\section{Biographical information}

William J. Ellison obtained the Ph. D. degree from Cambridge University, England. He is aat present Directeur de Recherche at the Centre National de Recherche Scientifique, France.
Jacques-Marie Moreau obtained the Ph. D. degree from the University of Bordeaux, France. He was professor at the university of Bordeaux until his retirement in 2005. 
> IM 06-8408 > 


\section{TABLE AND FIGURE CAPTIONS}

TABLE I VARIATION OF $C_{1}$ AND $C_{2}$ WITH FREQUENCY FOR A 3.6 COAXIAL PROBE FOR THE PERMITTIVITY RANGE $5 \leq \varepsilon ' \leq 100$ AND 5 $\leq \varepsilon^{\prime \prime} \leq 100$

Fig. 1 PARAMETRIZATION OF THE COMPLEX AdMitTANCE PLANE AT 100MHz.

Fig. 2 PARAMETRIZATION OF THE COMPLEX AdMitTANCE PLANE AT 1000MHz.

Fig.3 PARAMETRIZATION OF THE COMPLEX AdMITTANCE PlanE AT 2000MHz.

Fig. 4 PARAMETRIZATION OF THE COMPLEX AdMITTANCE PlANE AT 5000MHz

Fig. 5 SCATTER DIAGRAM ( $\varepsilon$ ' IS ABSCIISSA, $\varepsilon$ " IS ORDINATE) FOR THE VALIDITY OF THE LUMPED CAPACITY MODEL AT 1 GHZ. (OPEN SQUARES CORRESPOND TO PAIRS $\left(\varepsilon^{\prime}, \varepsilon^{\prime}\right)$ FOR WHICH THE MODEL ERROR IS $\left.<1 \%\right)$ 
ONE TABLE

\begin{tabular}{|c|c|c|}
\hline$G H z$ & $C_{1} p F$ & $C_{2} p F$ \\
\hline 0.1 & 0.597943 & 0.899251 \\
\hline 0.2 & 0.586221 & 0.899513 \\
\hline 0.3 & 0.562271 & 0.899963 \\
\hline 0.4 & 0.535866 & 0.900591 \\
\hline 0.5 & 0.498545 & 0.901392 \\
\hline 0.6 & 0.453240 & 0.990236 \\
\hline 0.7 & 0.400015 & 0.903505 \\
\hline 0.8 & 0.338888 & 0.904828 \\
\hline 0.9 & 0.270034 & 0.906322 \\
\hline 1.0 & 0.184453 & 0.909966 \\
\hline
\end{tabular}

TABLE I VARIATION OF $C_{1}$ AND $C_{2}$ WITH FREQUENCY FOR A 3.6 COAXIAL PROBE FOR THE PERMITTIVITY RANGE $5 \leq \varepsilon^{\prime} \leq 100$ AND $5 \leq \varepsilon^{\prime \prime} \leq 100$ 
FIVE FIGURES, ONE PER PAGE

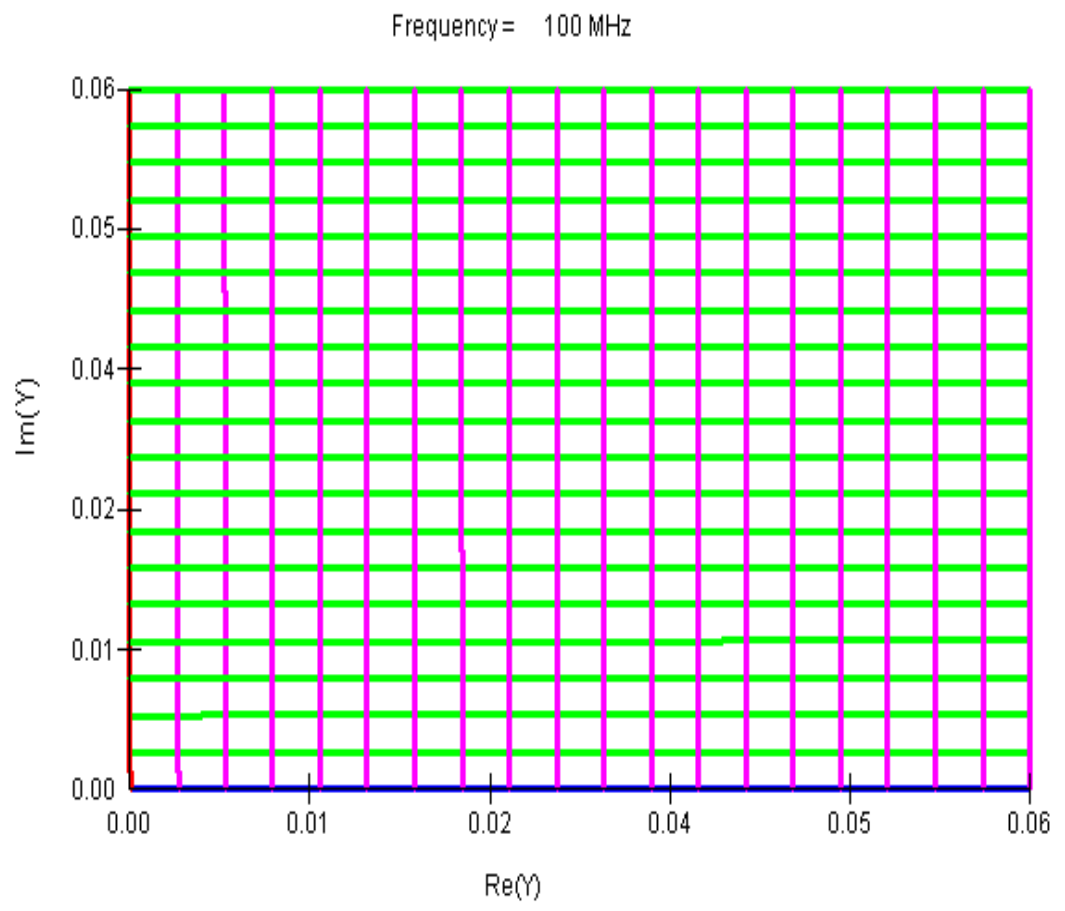

Fig. 1 PARAMETRIZATION OF THE COMPLEX ADMITTANCE PLANE AT 100MHz. 
Frequency $=1 . \mathrm{GHz}$

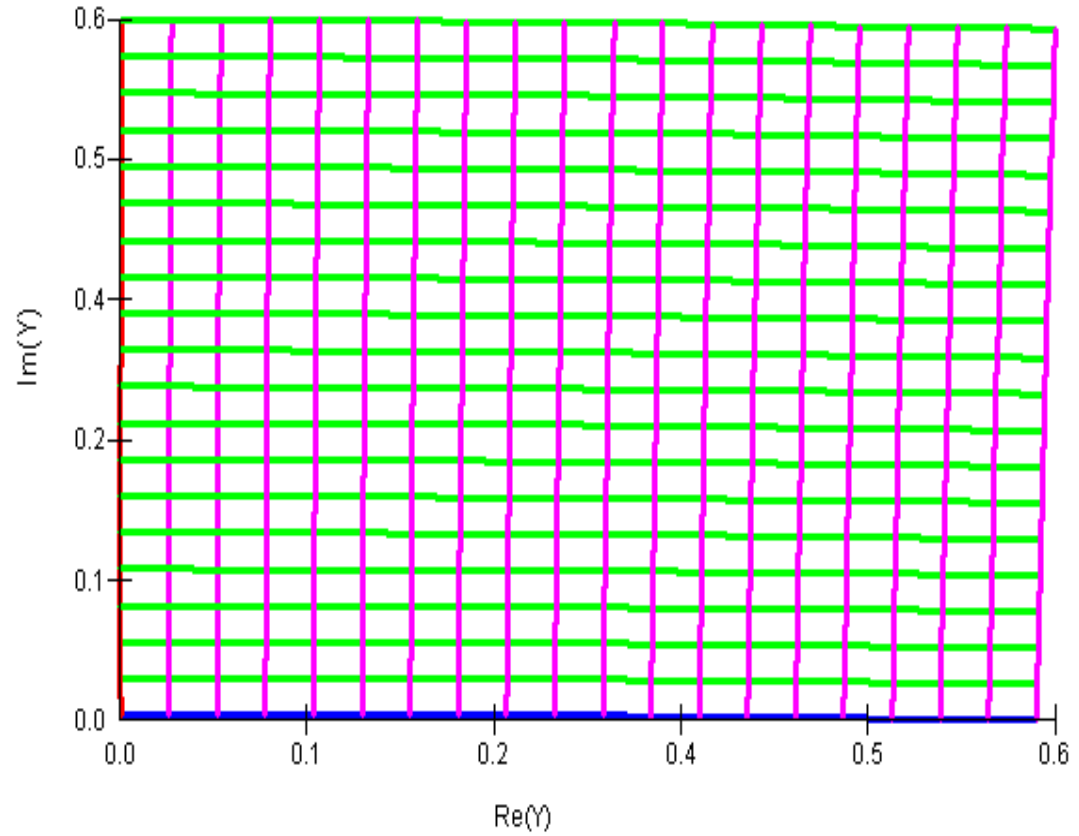

FIG. 2 PARAMETRIZATION OF THE COMPLEX ADMITTANCE PLANE AT 1000MHz. 


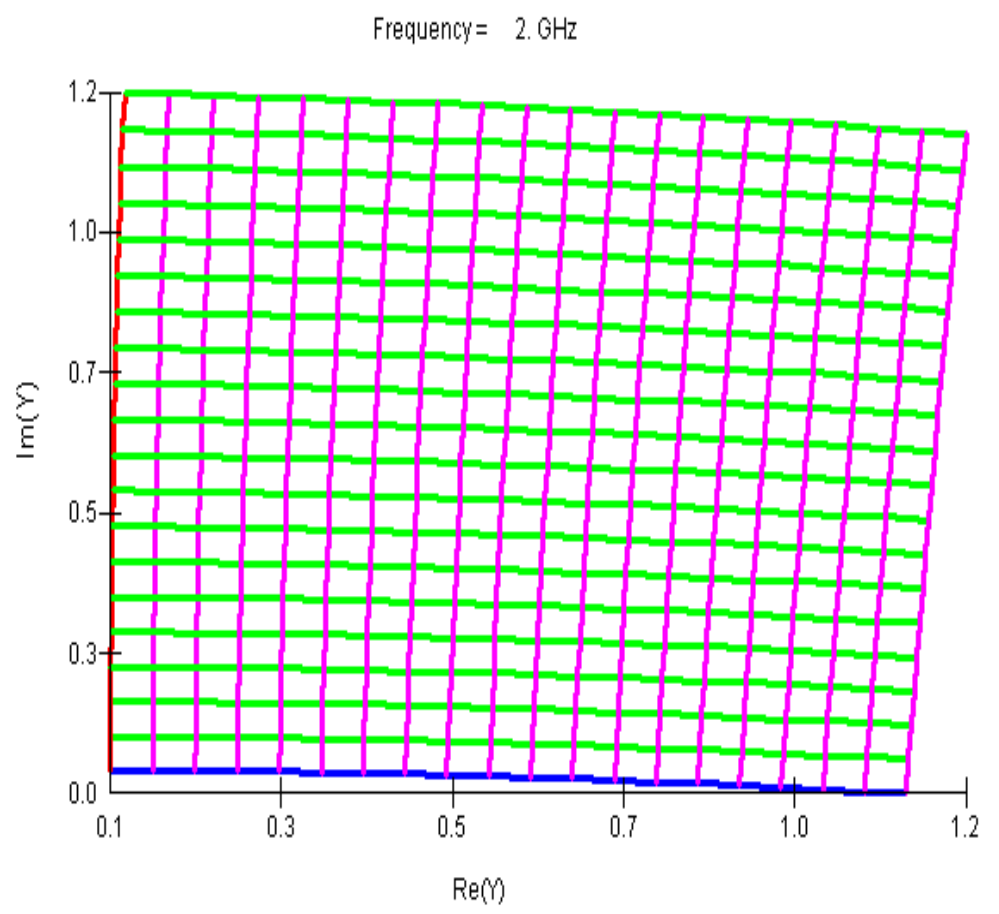

Fig.3 PARAmetrizAtion of THE COMPLEX ADMitTANCE PLANE AT 2000MHz. 
Frequency $=5 . \mathrm{GHz}$

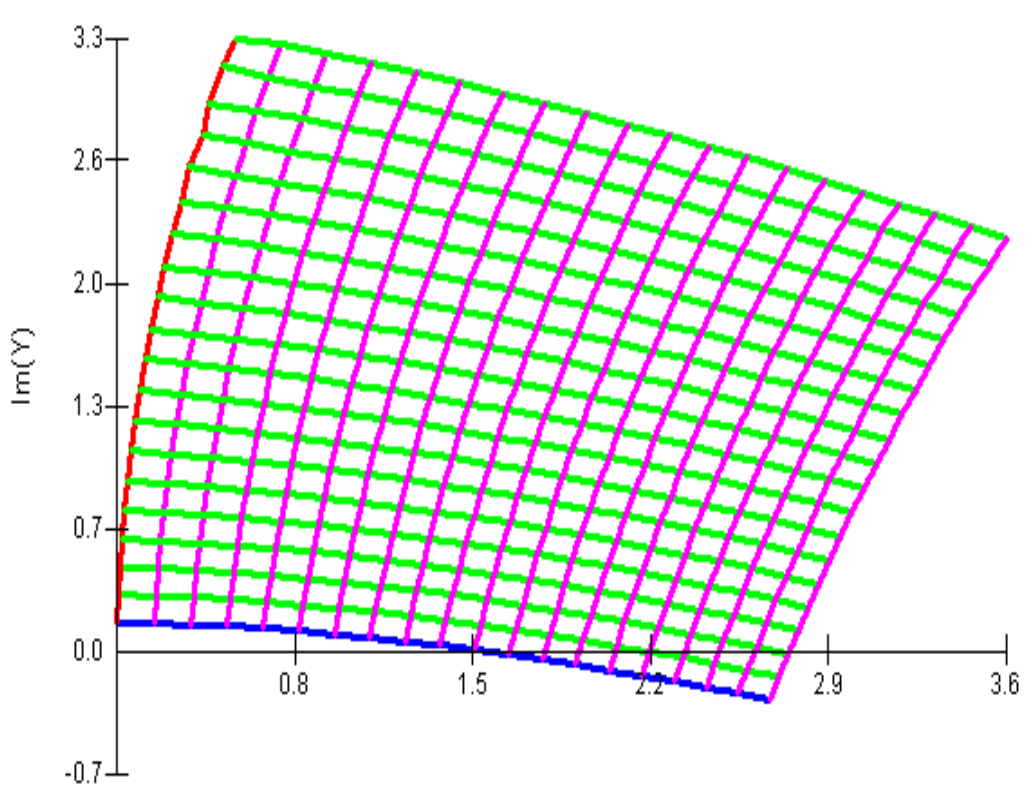

$\operatorname{Re}(\eta)$

FIG. 4 PARAMETRIZATION OF THE COMPLEX ADMITTANCE PLANE AT 5000MHz 


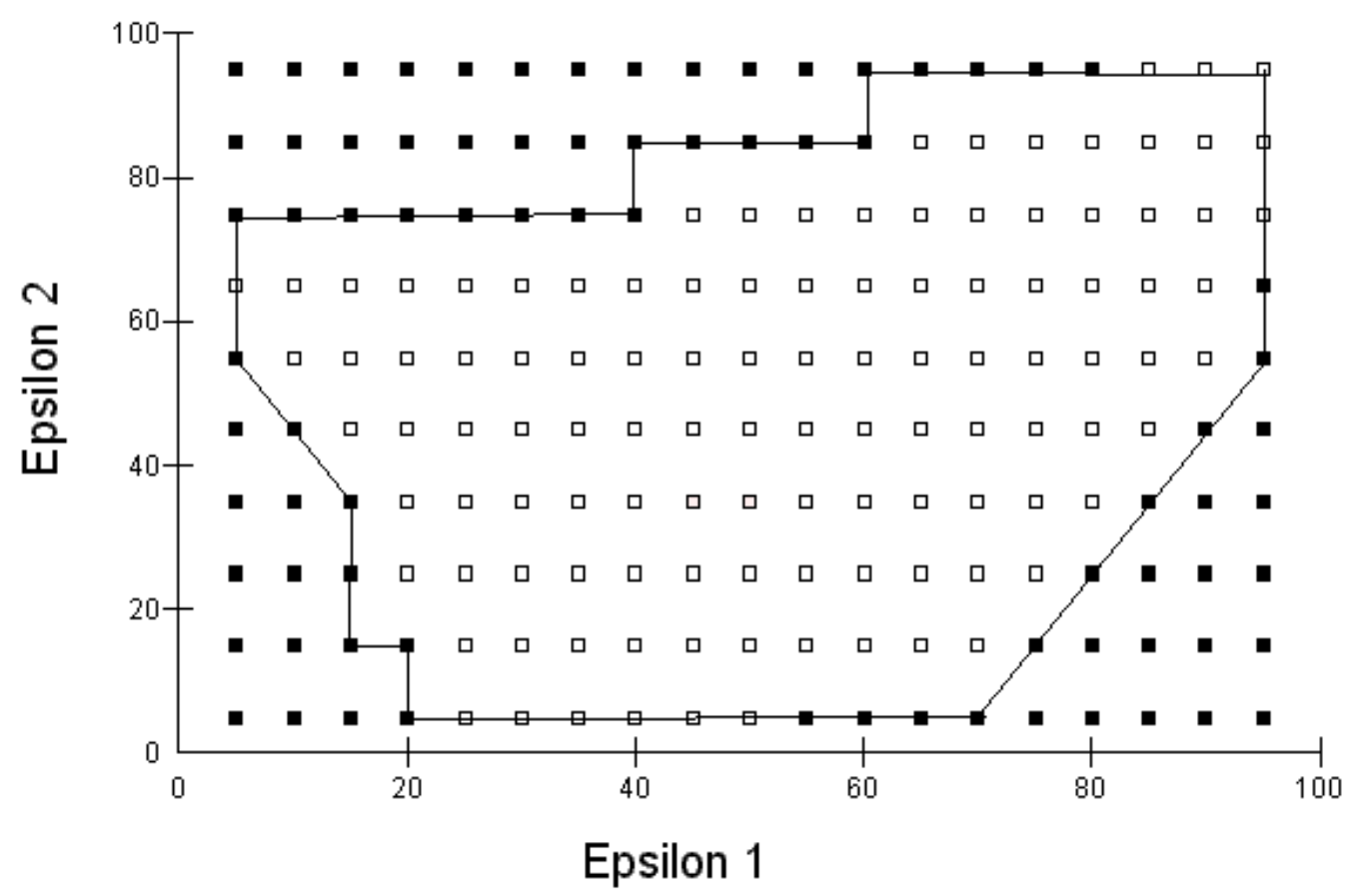

Fig. 5 SCATTER DIAGRAM ( $\varepsilon^{\prime}$ IS ABSCIISSA, $\varepsilon$ " IS ORDINATE) FOR THE VALIDITY OF THE LUMPED CAPACITY MODEL AT 1 GHz. (OPEN SQUARES CORRESPOND TO PAIRS $\left(\varepsilon^{\prime}, \varepsilon^{\prime}\right.$ ') FOR WHICH THE MODEL ERROR IS $<1 \%$ ) 